\title{
REBROTE Y ESTABILIDAD DE LA POBLACIÓN DE TALLOS EN EL PASTO Panicum maximum CV. 'MOMBAZA' COSECHADO EN DIFERENTES INTERVALOS DE CORTE
}

\author{
TURNOVER AND TILLER POPULATION STABILITY OF Panicum maximum CV. 'MOMBAZA' GRASS \\ HARVESTED AT DIFFERENT CUTTING INTERVALS
}

\author{
Omar Ramírez Reynoso ${ }^{1 \star}$, Sila Carneiro da Silva ${ }^{2}$, Alfonso Hernández Garay ${ }^{3}$, Javier F. Enríquez Quiroz ${ }^{4}$, Jorge \\ Pérez Pérez ${ }^{3 \dagger}$, Adrián R. Quero Carrillo ${ }^{3}$ y José G. Herrera Haro ${ }^{3}$
}

\begin{abstract}
${ }^{1}$ Unidad Académica de Medicina Veterinaria y Zootecnia en Costa Chica, Universidad Autónoma de Guerrero. Carretera Acapulco-Pinotepa Nacional Km 195. Cuajinicuilapa, Guerrero, México. Tel. 0174141 40783. ${ }^{2}$ Departamento de Zootecnia, Escoela Superior de Agricultura "Luiz de Queiroz"-Universidade de Sâo Paulo. Av. Pádua Días No 11. Piracicaba, Sâo Paulo, Brasil. ${ }^{3}$ Colegio de Postgraduados-Campus Montecillo. Km 36.5 Carretera México-Texcoco. 56230, Montecillo, Texcoco, Edo. de México. ${ }^{4}$ Campo Experimental Cotaxtla, Instituto Nacional de Investigaciones Forestales Agrícolas y Pecuarias. Álamos 29, Col. Limón de Guerrero. Isla, Veracruz, México.
\end{abstract}

*Autor para correspondencia (oramirez@colpos.mx).

\section{RESUMEN}

El objetivo del estudio fue determinar la densidad, tasa de rebrotación o recambio y estabilidad de la población de tallos del pasto Panicum maximum Jacq. cv. 'Mombaza', cosechado a intervalos de cortes (IC) de 3,5 y 7 semanas, durante un año, en las épocas de sequía (10-nov-2006 a 09-jun-2007) y lluvias (10-jun a 06-nov-2007). Los IC (tratamientos) se distribuyeron en 12 parcelas de $17.5 \mathrm{~m}^{2}$, mediante un diseño de bloques completos al azar con cuatro repeticiones. Las mayores densidades de tallos se registraron en el IC de 3 semanas durante la sequía (428 tallos $\mathrm{m}^{-2}$ ), y en los IC de 3 y 7 semanas durante las lluvias (413 y 394 tallos $\mathrm{m}^{-2}$, respectivamente). Hubo mayor dinámica de recambio de tallos durante la época de lluvias que en la de sequía, con mayores tasas de aparición (38.0 vs. 4.6 tallos/100 tallos $\mathrm{x}$ mes, respectivamente) y muerte de tallos (32.9 vs. 6.4 tallos/100 tallos $\mathrm{x}$ mes, respectivamente) y menores tasas de supervivencia (67.1 vs. 93.6 tallos/100 tallos $\mathrm{x}$ mes, respectivamente). Los IC de 3 y 5 semanas promediaron índices de estabilidad de la población de tallos próximos a uno, valores que indican estabilidad de la población, mientras que con el IC de 7 semanas este índice fue inferior a uno con inestabilidad de la población. Hubo menor dinámica de rebrote de tallos durante la sequía, con bajas tasas de aparición y muerte de tallos pero mayor supervivencia de la población, mientras que durante las lluvias se favoreció el rebrote. El pasto 'Mombaza' puede cosecharse cada 3 y 5 semanas sin comprometer la estabilidad de la población de tallos. Con el intervalo de cortes cada 7 semanas la recuperación frente a la muerte de tallos fue insuficiente, lo que propicia inestabilidad de la población.

Palabras clave: Panicum maximum, rebrote de tallos, supervivencia de tallos, estabilidad de la población.

\section{SUMMARY}

The objective of this experiment was to evaluate tiller density, tiller turnover and tiller population stability of Panicum maximum Jacq. cv. 'Mombaza' grass, harvested at three cutting intervals (CI; 3, 5 and 7 weeks) throughout a 12-month period comprising the "dry season"
(Nov 10, 2006 to Jun 09, 2007) and the "rainy season" (Jun 10-to -Nov $06,2007)$. Treatments $(\mathrm{CI})$ were assigned to 12 experimental units $(17.5$ $\mathrm{m}^{2}$ plots) according to a complete randomised block design with four replications. Tiller population densities were greater for the 3-weeks CI during the dry season (428 tillers $\left.\mathrm{m}^{-2}\right)$ and for the 3 and 7-weeks CI during the rainy season (413 and 394 tillers $\mathrm{m}^{-2}$, respectively). During the rainy season tiller turnover process was more intense and characterised by the highest rates of tiller appearance $(38.0 \mathrm{vs} .4 .6$ tillers 100/tillers $\mathrm{x}$ month) and death (32.9 vs. 6.4 tillers/100 tillers $\mathrm{x}$ month), and lowest survival (67.1 vs. 93.6 tillers 100/tillers $\mathrm{x}$ month). Cutting intervals of 3 and 5 -weeks averaged population stability indexes close to one, but the 7-weeks CI was less than one. During the dry season there was a lower tiller turnover, with lower tiller appearance and death rates but higher tiller population stability, than during the rainy season when the tiller turnover increased. 'Mombaza' grass may be harvested every 3 and 5 weeks without compromising its tiller population stability. The 7-weeks CI did not allow an efficient replacement of dead tillers thus leading to instability of the tiller population.

Index words: Panicum maximum, tiller turnover, tiller survival, tiller population stability.

\section{INTRODUCCIÓN}

En los sistemas de producción animal basados en praderas, tanto la producción de forraje como la persistencia de éstas son factores importantes para la sostenibilidad de esos sistemas. La persistencia de las praderas depende de la capacidad de las plantas para renovar los tallos muertos y mantener estable la densidad poblacional de tallos, lo que está directamente determinado por el efecto combinado de los patrones estacionales en los procesos de aparición, muerte y supervivencia de tallos. Existen diferencias entre especies y cultivares para lograr la estabilidad de la densidad poblacional de tallos y favorecer la persistencia 
de las praderas. Phleum pratense posee un mecanismo de persistencia considerado como reproductivo, debido a que la rebrotación de tallos proviene de la base de los tallos cosechados y su meristemo apical eliminado, que ocurre durante la etapa de floración (Jewiss, 1966; citado por Matthew et al., 2000). En cambio, Paspalum notatum puede mantener una densidad de tallos estable durante cuatro años debido a que sus tallos son de vida larga, con bajas tasas de aparición y muerte de tallos (Hirata y Pakiding, 2001). En Lolium perenne cv. 'Nui', el recambio o rebrotación de tallos presenta variaciones en las tasas de aparición y muerte de tallos que dependen del manejo de la pradera y la estación del año; la más alta tasa de aparición de tallos ocurre después de la interrupción del crecimiento, durante la etapa reproductiva al inicio de verano, lo que favorece el incremento en la densidad de tallos (Hernández-Garay et al., 1997). En contraste, Lolium perenne cv. 'Ruanui' tiene un mecanismo de persistencia considerado como vegetativo, debido a que la rebrotación proviene de tallos sin florecer, a una tasa constante (Matthew et al., 1993).

Las variaciones estacionales en las tasas de aparición, muerte y supervivencia de tallos, son importantes para comprender los mecanismos involucrados en la persistencia y rebrotación (recambio) de tallos en las praderas. Sin embargo, los análisis independientes de estas tasas no indican la estabilidad de la población de tallos en determinada época; es decir, si la aparición de tallos con respecto a su muerte es suficiente para mantener la población en equilibrio. La estabilidad de la población de tallos de un pasto puede calcularse por medio de un índice que se obtiene de la relación entre los procesos de supervivencia y aparición de tallos, y permite un análisis integral de las variaciones de la población (Bahmani et al., 2003).

El cultivar 'Mombaza' de Panicum maximum Jacq. es originario de Tanzania, África, que fue introducido a Brasil en 1982 y luego liberado en 1993 por el Centro Nacional de Pesquisa de Gado de Corte (CNPGC) como un cultivar de alta producción de forraje y adaptabilidad en diversos ambientes tropicales (Souza, 1999). Recientemente fue introducido a México como alternativa para mejorar la productividad de las praderas tropicales (Enríquez et al., 1999). En este país, Ramírez et al. (2009) reportaron que la acumulación anual de forraje fue de 12 200, 19800 y $24300 \mathrm{~kg} \mathrm{MS} \mathrm{ha}^{-1} \mathrm{año}^{-1}$ cuando las praderas se cosecharon cada 3, 5 y 7 semanas, respectivamente, con $83 \%$ de esta acumulación durante la época de lluvias, y la proporción de hojas en el forraje acumulado era de 75, 65 y $56 \%$ para los mismos de intervalos de corte. Con respecto a la formación de órganos, en este cultivar la generación de hojas fue mayor con cortes cada 3 semanas que con intervalos de 5 y 7 semanas; la elongación de tallos y hojas fue mayor durante la época de lluvias; hubo mínima senescencia de hojas durante la sequía, y fue más alta con cortes cada 7 semanas durante las lluvias (Ramírez et al., 2010). En estos estudios no se evaluó la dinámica de recambio de tallos ni su impacto en la estabilidad de la población, a pesar de su importancia para hacer recomendaciones específicas de manejo para este cultivar.

Por lo anterior, el objetivo de esta investigación fue determinar la densidad, tasa de rebrotación y estabilidad de la población de tallos del pasto Panicum maximum Jacq. cv. 'Mombaza', en respuesta a tres intervalos de corte, en las épocas de sequía y lluvias, durante un año.

\section{MATERIALES Y MÉTODOS}

\section{Localización y duración del estudio}

El estudio se llevó a cabo en el rancho "Aztlan" (16 43’26” LN y $99^{\circ} 07^{\prime} 24^{\prime \prime}$ LO), ubicado en el municipio de Florencio Villarreal, Guerrero, México, durante el periodo de noviembre del 2006 a noviembre del 2007. El clima del sitio es $A_{\mathrm{o}}$, que corresponde a cálido subhúmedo con lluvias en verano (García, 1981), con temperatura y precipitación media anual de $27^{\circ} \mathrm{C}$ y lluvia anual de $1097 \mathrm{~mm}$, a $40 \mathrm{~m}$ de altitud (CONAGUA, 2008). Los datos mensuales de precipitación y temperaturas máximas, media y mínima, ocurridos durante el periodo experimental (Figura 1), se obtuvieron en la estación climatológica del Centro de Estudios de Bachillerato Tecnológico y Agropecuario 151, ubicado a $3 \mathrm{~km}$ del área experimental.

\section{Manejo de praderas}

En un área de $600 \mathrm{~m}^{2}(15 \times 40 \mathrm{~m})$, se establecieron cuatro parcelas de $70 \mathrm{~m}^{2}(10 \times 7 \mathrm{~m})$, con $2 \mathrm{~m}$ de separación entre sí. La siembra se hizo manualmente, $15 \mathrm{~d}$ después del inicio de las lluvias (03-jul-2006), mediante un sistema de labranza mínima que consistió en la aspersión de $3 \mathrm{~L} \mathrm{ha}^{-1}$ de un herbicida sistémico (i.a., glifosato $40.5 \%$ ), un día antes de la siembra, para eliminar la biomasa emergida. La densidad de siembra fue de $5 \mathrm{~kg} \mathrm{ha}^{-1}$ de semilla comercial con valor cultural de $35 \%$, distribuida a $50 \mathrm{~cm}$ entre hileras y plantas. Cuatro meses después de la siembra las parcelas se dividieron en tres unidades experimentales de $17.5 \mathrm{~m}^{2}$ (5 x $3.5 \mathrm{~m}$ ), en las que se distribuyeron los tratamientos en un diseño de bloques completos al azar, que consistieron en tres intervalos de corte (IC) a $5 \mathrm{~cm}$ de altura: 3,5 y 7 semanas, combinados con dos épocas de estudio: sequía (10-nov2006 a 09-jun-2007) y lluvias (10-jun a 06-nov-2007). Al inicio de cada época se hizo un corte de uniformidad a 5 $\mathrm{cm}$ de altura. Durante el estudio, la maleza de hoja ancha se controló químicamente con 2-4 D + Picloram ${ }^{\circledR}$ a una dosis de $2 \mathrm{~L} \mathrm{ha}^{-1}$. 


\section{Variables estudiadas}

Densidad y tasas de aparición y muerte de tallos. El número de tallos aparecidos, muertos y total se determinó en cada unidad experimental en un área de $1 \mathrm{~m}^{2}$, en la cual también se registró el número de plantas; además, se seleccionó una planta representativa (de altura promedio) de cada unidad experimental, en la que se marcaron los tallos vivos (01-dic-2006) con anillos de cable de un color, que se consideraron como población inicial. Posteriormente, con frecuencia mensual durante un año, los tallos nuevos se marcaron con anillos de diferente color para cada generación, y los tallos muertos se registraron y se les retiró el anillo. Estos valores se multiplicaron por el número de plantas $\mathrm{m}^{-2}$, y se estimó la densidad de tallos (DT; tallos $\mathrm{m}^{-2}$ ) y sus respectivas tasas de aparición (TAT) y muerte (TMT) (\%), mediante la metodología sugerida por Carvalho et al. (2000), de acuerdo con las ecuaciones 1, 2 y 3.

DT = Número total de tallos vivos existentes en cada muestreo

$\mathrm{TAT}=$ (Número de tallos nuevos $/ \mathrm{DT}$ del muestreo anterior) 100

$\mathrm{TMT}=$ (Número de tallos muertos / DT del muestreo anterior) 100

La tasa de supervivencia de tallos (TST) se obtuvo de manera indirecta por medio de la Ec. 4 y el índice de estabilidad de la población de tallos (Pf/Pi) se obtuvo con la Ec. 5 sugerida por Bahmani et al. (2003).

$$
\begin{aligned}
& \mathrm{TST}=100-\mathrm{TMT} \\
& \mathrm{Pf} / \mathrm{Pi}=\mathrm{TST}(1+\mathrm{TAT})
\end{aligned}
$$

donde Pf/Pi es el cociente de la población actual o final de tallos (Pf) entre la población inicial de tallos o anteriormente observada (Pi), y su valor indica el efecto combinado de la aparición y muerte de tallos con relación a la población total, para un periodo determinado. La interpretación de este índice se basa en que los valores iguales o próximos a uno (0.95 a 1.05) indican una población de tallos estable, en la que el número de tallos prácticamente no varía, aunque es resultado de un equilibrio dinámico. Los valores inferiores a uno $(<0.95)$, indican inestabilidad negativa, donde la población de tallos tiende a disminuir a través del tiempo, y los valores superiores a uno (> 1.05) representan la condición inversa (Bahmani et al., 2003).

\section{Análisis de datos}

El análisis estadístico de los datos se hizo con el procedimiento PROC MIXED del paquete estadístico SAS (SAS Institute, 2002). Para seleccionar la matriz de varianza y covarianza se utilizó el criterio de información de Akaike (Wolfinger, 1993). Los efectos de intervalo de cortes, época del año y sus interacciones se consideraron fijos, y el efecto de bloques se consideró aleatorio. Las medias de las causas de variación (tratamientos, época del año y su interacción) se estimaron con el procedimiento LSMEANS, y la comparación entre ellas se hizo por medio de la probabilidad de la diferencia (PDIFF) con la prueba de " $\mathrm{t}$ " de Student, a un nivel de significancia de $5 \%$. Para el análisis de la densidad de tallos se utilizó como covariable la población inicial (primera densidad registrada, posterior al corte de uniformidad) de cada época. La demografía de tallos durante el estudio aquí se presenta mediante un diagrama por cada intervalo de corte, con base en los estadísticos descriptivos (promedios) de cada generación de tallos.

\section{RESULTADOS Y DISCUSIÓN}

\section{Demografía de tallos}

Los diagramas de la demografía de tallos (Figura 2) muestran la supervivencia a través del tiempo, de cada generación que apareció entre mediciones sucesivas, y su contribución a la población total de tallos en un momento determinado. Estos diagramas proveen una visión general del conjunto de datos y facilitan la comprensión y discusión de los resultados.

\section{Densidad y tasas de aparición y muerte de tallos}

Hubo efecto del intervalo de corte, IC $(\mathrm{P}=0.0058)$ y de la interacción ( $I C \mathrm{x}$ época; $\mathrm{P}=0.0385$ ) en la densidad de tallos; efecto de época $(P<0.0001)$ y de la interacción (IC x época; $\mathrm{P}=0.0326)$ en la tasa de aparición de tallos; y efecto de época $(\mathrm{P}<0.0001)$ y de IC $(\mathrm{P}=0.0385)$ en la tasa de muerte de tallos (Cuadro 1). La densidad de tallos durante la época de sequía fue 26 y $42 \%$ mayor en el IC de 3 semanas que en los de 5 y 7 semanas, respectivamente, sin diferencia entre estos últimos IC. Durante la época de lluvias la densidad de tallos en los IC de 3 y 7 semanas fueron similares, pero 24 y $18 \%$ mayores respectivamente que la observada con el IC de 5 semanas. Hubo mayor tasa de recambio de tallos durante la época de lluvias que en la de sequía. La tasa de aparición de tallos en la época de sequía fue 211 y $190 \%$ mayor en el IC de 3 semanas que en los IC de 5 y 7 semanas, respectivamente, sin diferencia entre estos últimos. Durante la época de lluvias la tasa de aparición de tallos con el IC de 7 semanas fue $44 \%$ mayor que con el de 5 semanas, mientras que con el de 3 semanas el valor de esta tasa fue intermedio. La tasa de muerte de tallos fue 414 $\%$ mayor durante la época de lluvias que en la de sequía. Independientemente de la época, la tasa de muerte de tallos 
Cuadro 1. Densidad y tasas de aparición y muerte de tallos del pasto 'Mombaza', por época del año, cosechado a diferentes intervalos de corte.

\begin{tabular}{cccc}
\hline IC & Sequía & Lluvias & Promedio \\
\hline 3 & & Densidad (tallos $\left.\mathbf{~ m}^{-2}\right)$ & $420 \mathrm{~A}$ \\
5 & $428 \mathrm{~A}, \mathrm{a}$ & $413 \mathrm{~A}, \mathrm{a}$ & $336 \mathrm{~B}$ \\
7 & $339 \mathrm{~B}, \mathrm{a}$ & $333 \mathrm{~B}, \mathrm{a}$ & $348 \mathrm{~B}$ \\
Promedio & $302 \mathrm{~B}, \mathrm{~b}$ & $394 \mathrm{~A}, \mathrm{a}$ & 22.9 \\
EEM & 356 & 10.0 & 17.0 \\
& 10.0 & Tasa de aparición (tallos/100 tallos x mes) & 24.0 \\
3 & $8.4 \mathrm{~A}, \mathrm{~b}$ & $37.5 \mathrm{AB}, \mathrm{a}$ & \\
5 & $2.7 \mathrm{~B}, \mathrm{~b}$ & $31.3 \mathrm{~B}, \mathrm{a}$ & $18.3 \mathrm{~B}$ \\
7 & $2.9 \mathrm{~B}, \mathrm{~b}$ & $45.2 \mathrm{~A}, \mathrm{a}$ & $16.4 \mathrm{~B}$ \\
Promedio & $4.6 \mathrm{~b}$ & $38.0 \mathrm{a}$ & $24.3 \mathrm{~A}$ \\
EEM & 0.78 & 3.57 & 1.71 \\
\hline
\end{tabular}

Medias con letras mayúsculas iguales en cada columna de cada variable, no son estadísticamente diferentes (PDIFF, 0.05). Medias con letras minúsculas iguales en cada lhilera, no son estadísticamente diferentes (PDIFF, 0.05). IC = intervalo de cortes, en semanas; EEM = error estándar de la media.

con el IC de 7 semanas fue 48 y $33 \%$ mayor que con los de 5 y 3 semanas, respectivamente.

En praderas cosechadas cada 3 y 5 semanas, la densidad de tallos fue mayor al final del estudio que la densidad inicial, en tanto que en praderas cosechadas cada 7 semanas ocurrió lo contrario (Figura 2). Estos resultados fueron consecuencia de diferentes dinámicas de recambio de tallos (tasas de aparición y muerte de tallos) de las plantas, inducidas por las condiciones ambientales de las épocas y de los IC. Probablemente esto haya sido la manifestación de mecanismos de persistencia y colonización del área, ya que eran praderas nuevas con baja densidad de plantas. La mayor dinámica de recambio de tallos ocurrida durante la época de lluvias se atribuye a que en esta época hubo mejores condiciones ambientales (Figura 1), principalmente de precipitación.

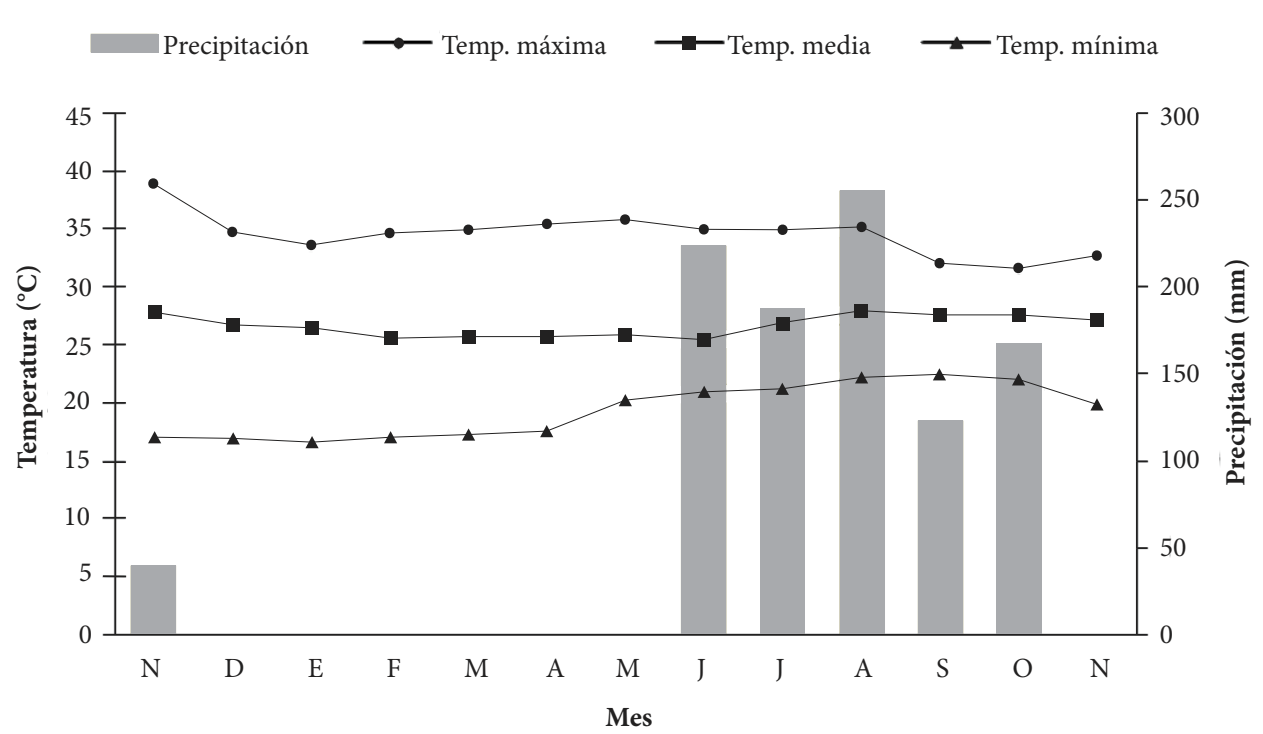

Figura 1. Datos mensuales de precipitación pluvial y temperaturas máxima, media y mínima, durante el periodo nov-2006 a nov-2007, en el Municipio de Florencio Villarreal, Guerrero, México. Fuente: Centro de Bachillerato Tecnológico Agropecuario, Núm. 151. 
Durante la época de sequía la mayor densidad de tallos registrada con el IC de 3 semanas, fue consecuencia de la mayor tasa de aparición de tallos (Cuadro 1), debido a que estos intervalos de cosecha cortos e intensos, favorecen la cantidad y calidad de luz que penetra a la base del dosel. Estas condiciones promueven la activación de yemas axilares (Matthew et al., 2000), localizadas en la base de los tallos remanentes y en la corona de las plantas (Carvalho et al., 2000); y estas yemas axilares son las que originan nuevos tallos. Contrariamente, los IC de 5 y 7 semanas favorecieron una mayor altura de plantas, con 23 y $30 \mathrm{~cm}$, respectivamente, como indicaron Ramírez et al. (2009), posiblemente porque permiten interceptar mayor cantidad de luz y disminuir la cantidad y calidad de luz que penetró a la base del dosel, con la consecuente inhibición de la aparición de tallos (Alexandrino et al., 2004). De manera similar, es posible que la menor radiación recibida en la base del dosel con el IC de 7 semanas, haya favorecido la muerte de tallos pequeños, como propusieron Carvalho et al. (2000) y con ello propicio la mayor tasa de muerte en este IC (Cuadro 1). Esta dinámica puede explicar, aunque sin evidencia estadística, que al final de esta época la cantidad de tallos en las praderas cosechadas cada 3, 5 y 7 semanas, aumentara, se mantuviera y disminuyera, respectivamente, con respecto a la densidad inicial (Figura 2).

La mayor densidad de tallos registrada en los IC de 3 y 7 semanas durante las lluvias, se debió a que la magnitud de la diferencia (6.1 y $6.5 \%$, respectivamente) entre sus respectivas tasas de aparición y muerte de tallos de esta época, fue mayor que la diferencia $(2.7 \%)$ registrada con el IC de 5 semanas (Cuadro 1). Si bien los intervalos de cosecha largos aumentan la competencia por luz durante el periodo de rebrote e inhiben el amacollamiento, en estas condiciones las praderas presentan menores densidades de tallos como afirmaron varios autores (Deregibus et al., 1985; Matthew et al., 2000; Lemaire, 2001). Sin embargo, en este estudio la superioridad del IC de 7 semanas en la tasa de aparición de tallos sobre el de 5 semanas, es un resultado que podría atribuirse a que con el IC de 7 semanas las praderas tuvieron mayor tasa de aparición de hojas (Ramírez et al., 2010), y la aparición de hojas determina el potencial de aparición de tallos porque en cada axila de hoja hay una yema capaz de producir un tallo (Skinner y Nelson, 1994). La mayor tasa de muerte de tallos ocurrida con el IC de 7 semanas (Cuadro 1), se debió a que con plantas más altas $(111 \mathrm{~cm})$ y consecuentemente de tallos más altos, la cantidad de meristemos apicales localizados arriba del nivel de cosecha, también debio ser mayor como observaron Lemaire y Chapman (1996), lo que pudo propiciar la eliminación de meristemos apicales y la muerte de tallos al momento de la cosecha, según reportaron Cecato et al. (2000). Esta dinámica propició que al final de las lluvias la densidad de tallos aumentara con respecto a la densidad inicial de esta época, en todos los IC (Figura 2).

Un estudio efectuado con este mismo cultivar, Adese et al. (2004) reportaron ausencia de efecto del intervalo de corte (correspondientes a intervalos de la aparición de 2, 3 y 4 hojas por tallo), en las tasas de aparición y muerte de tallos basales; en cambio, fueron afectadas por la intensidad de cosecha $(25$ y $50 \mathrm{~cm}$ ). Diversos estudios efectuados en condiciones de pastoreo han reportado diferencias en la densidad de tallos entre épocas del año (Santos et al., 1999), entre intensidades de cosecha (25 y $40 \mathrm{~cm})$, a intervalos de pastoreo de $35 \mathrm{~d}$ (Barbosa et al., 2002); también se ha reportado falta de efecto de los intervalos en pastoreos realizados cada 28, 38 y 48 d (Santos et al., 2004). Por su parte, Cândido et al. (2005) registraron mayor densidad de tallos con menores periodos de descanso que con descansos

Cuadro 2. Tasa de sobrevivencia e índice de estabilidad de la población de tallos del pasto 'Mombaza', por época del año, cosechado a diferentes intervalos de corte.

\begin{tabular}{cccc}
\hline IC & Sequía & Lluvias & Promedio \\
\hline 3 & & Tasa de sobrevivencia (tallos/100 tallos x mes) & $81.68 \mathrm{~A}$ \\
5 & 94.82 & 68.55 & $83.60 \mathrm{~A}$ \\
7 & 95.80 & 71.40 & $75.65 \mathrm{~B}$ \\
Promedio & 90.07 & 61.22 & 1.710 \\
EEM & $93.56 \mathrm{a}$ & $67.05 \mathrm{~b}$ & $0.9840 \mathrm{~A}$ \\
& 1.043 & 3.306 & $0.9600 \mathrm{AB}$ \\
3 & & Índice de estabilidad de la población & $0.9058 \mathrm{~B}$ \\
5 & 1.0285 & 0.9395 & 0.01758 \\
\hline
\end{tabular}

Medias con letras mayúsculas iguales en cada columna de cada variable, no son estadísticamente diferentes (PDIFF, 0.05). Medias con letras minúsculas iguales en cada línea de cada variable, no son estadísticamente diferentes (PDIFF, 0.05). IC = intervalo de cortes, en semanas; EEM = error estándar de la media. 

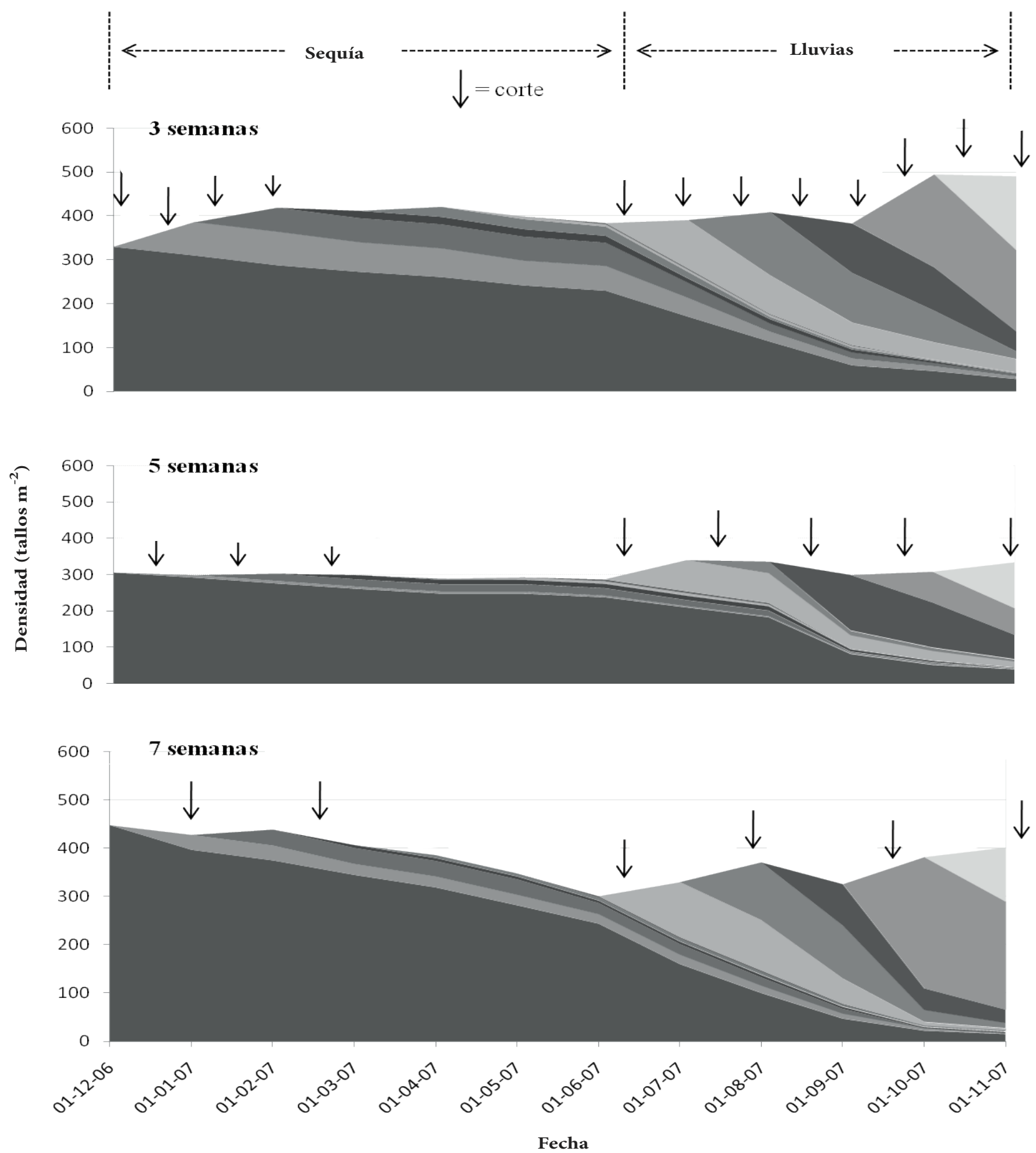

Figura 2. Cambios mensuales en la demografía de tallos del pasto Panicum maximum cv. 'Mombaza' cosechado a diferente intervalo de cortes (3, 5 y 7 semanas). Cada tono de sombra representa la dinámica poblacional dentro de la misma generación de tallos. 
más prolongados. La diversidad de condiciones en las que se desarrollaron esos trabajos no permiten que se comparen sus resultados con los de este estudio, en particular por diferencias en la intensidad y método de cosecha. Asimismo, tal como se indicó en la introducción, debido a esa diversidad no se cuenta aún con información que permita realizar recomendaciones de manejo específicas para este cultivar.

\section{Tasas de sobrevivencia e índices de estabilidad de la población de tallos}

La tasa de sobrevivencia de tallos presentó efecto de IC (P $=0.0385)$ " y de época $(\mathrm{P}<0.0001)$. El índice de estabilidad de la población de tallos mostró efecto de IC $(\mathrm{P}=0.0490)$ y de época $(\mathrm{P}=0.0163)$ (Cuadro 2). La tasa de supervivencia de tallos fue $40 \%$ mayor durante la época de sequía que durante la de lluvias. Independientemente de la época los IC de 3 y 5 semanas tuvieron 8 y $11 \%$ mayor tasa de supervivencia que el de 7 semanas. El índice de estabilidad de la población de tallos durante la sequía fue mayor y próximo de 1 (0.98), en tanto que en la época de lluvias fue inferior a 1 (0.92). El índice de estabilidad con el IC de 3 semanas dio un valor próximo a uno (0.98), mayor que con el IC de 7 semanas que fue inferior a uno (0.90), mientras que con el IC de 5 semanas tuvo un valor intermedio, con un índice próximo a uno (0.96).

Las mayores tasas de supervivencia de tallos (Cuadro 2) durante la sequía fueron resultado directo de las bajas tasas de muerte (Cuadro 1), lo que sugiere que la supervivencia de tallos puede ser un mecanismo de adaptación utilizado por las plantas para mantener la persistencia de tallos en condiciones de déficit hídrico. Durante las lluvias, la menor tasa de supervivencia de tallos (Cuadro 2) aunada a las altas tasas de aparición (Cuadro 1), favorecieron la tasa de recambio de tallos y el incremento de sus densidades de población (Figura 2), posiblemente como un mecanismo para colonizar el área cuando hubo condiciones ambientales apropiadas y baja densidad de plantas, como en este estudio.

El análisis del índice de estabilidad de la población de tallos mostró que las praderas de 'Mombaza' fueron más estables en sequía que durante las lluvias (Cuadro 2), debido a que en condiciones de deficiencia hídrica las plantas presentaron menor dinámica de recambio de tallos (Cuadro 1). Esta menor dinámica de recambio posiblemente redujo la competencia por factores para el crecimiento, y en consecuencia la persistencia de tallos fue mayor (Cuadro 2). Independientemente de la época, los IC de 3 y 5 semanas presentaron índices de estabilidad similares y con valores próximos a uno (Cuadro 2), lo que sugiere que con esos IC la población de tallos tendió a permanecer estable. Por el contrario el IC de 7 semanas presentó menor índice de estabilidad con valor inferior a uno (Cuadro 2), lo que sugiere que la población de tallos se mantuvo inestable. La población de tallos tendió a disminuir (Figura 2) debido a la mayor tasa de muerte de tallos (Cuadro 1) y menor tasa de supervivencia (Cuadro 2), lo que puede implicar la degradación de la pradera.

Estos resultados difieren de los de Morais et al. (2006), quienes no encontraron diferencias en la tasa de sobrevivencia de tallos entre épocas del año, al estudiar el amacollamiento de la especie Brachiaria brizantha durante diez meses, en praderas mantenidas a $20 \mathrm{~cm}$ de altura mediante pastoreo continuo; la discrepancia probablemente se deba a diferencias en el método y en la intensidad de cosecha aplicados. Un análisis del índice de estabilidad de la población efectuado en praderas de Lolium perenne L. mostró diferencias entre cultivares y entre épocas del año, cuando fueron sometidas a estímulos externos como fertilización nitrogenada e irrigación (Bahmani et al., 2003). Por su parte, las praderas de clima cálido plantadas con Brachiaria brizanta cv. 'Marandu', cosechadas a dos alturas $(15$ y $30 \mathrm{~cm})$ y tres intervalos de corte (aparición de tres, cuatro y cinco hojas por tallo), mostraron índices de estabilidad de la población superiores a uno en todos los intervalos de corte, pero que luego tendieron a disminuir conforme el periodo experimental avanzó de la época de sequía a la de lluvias (Difante et al., 2008); esta tendencia es similar a las diferencias aquí observadas entre épocas.

\section{CONCLUSIONES}

Las plantas del pasto 'Mombaza' presentaron menor dinámica de recambio de tallos durante la sequía, con bajas tasas de aparición y muerte de tallos y mayor supervivencia de la población, mientras que durante las lluvias hubo mayor dinámica de recambio, con altas tasas de aparición y muerte de tallos y menor supervivencia de la población. La cosecha del pasto 'Mombaza' cada 3 y 5 semanas no comprometió la estabilidad de la población, debido a que la aparición y supervivencia de tallos permitieron mantener la densidad frente a la muerte de tallos. El intervalo de corte cada 7 semanas comprometió la estabilidad de la población porque la reposición de tallos muertos fue insuficiente.

\section{BIBLIOGRAFÍA}

Adese B, D N Junior, V P B Euclides, S C Da Silva, G D S Difante, A M Zanine, D B Montagner, K S Pena, M C T D Silveira, D D M Rezende, R A Barbosa (2004) Dinâmica do perfilhamento em capim-mombaça submetido a freqüências e intensidades de desfolhação. In: Symposium on Grassland Ecophysiology and Grazing Ecology. E Pizarro, P C F Carvalho, S C Da Silva (eds). UFPR. Curitiba, Brazil. 2004:CD-ROM.

Alexandrino E, D N Júnior, P R Mosquim, A J Regazzi, F C Rocha (2004) Características morfogênicas e estruturais na rebrotação da Brachiaria brizantha cv. Marandu submetida a três doses de 
nitrogênio. Rev. Bras. Zoot. 33:1372-1379.

Bahmani I, E R Thom, C Matthew, R J Hooper, G Lemaire (2003) Tiller dynamics of perennial ryegrass cultivars derived from different New Zealand ecotypes: effects of cultivar, season, nitrogen fertilizer, and irrigation. Aust. J. Agric. Res. 54:803-817.

Barbosa A R, J D Nascimento, B V P Euclides, J A Regazzi, M D Fonseca (2002) Características morfogênicas e acúmulo de forragem do capim-Tanzânia (Panicum maximum Jacq. cv. Tanzânia) em dois resíduos forrageiros pós-pastejo. Rev. Bras. Zoot. 31:583-593.

Cândido D M J, M C A Gomide, E Alejandrino, J A Gomide, E W Pereira (2005) Morfofisiologia do dossel de Panicum maximum cv. Mombaça sob lotação intermitente com três períodos de descanso. Rev. Bras. Zoot. 34:406-415.

Carvalho B C A, S C Da Silva, F A Sbrissia, M L F Pinto, A R Carnevalli, L J Fagundes, S C G Pedreira (2000) Demografia do perfilhamento e taxas de acúmulo de matéria seca em capim 'Tifton 85 ' sob pastejo. Sci. Agric. 57:591-600.

Cecato U, A O M Machado, E N Martins, L A F Pereira, M A A F Barbosa, G T Santos (2000) Avaliação da produção e de algumas características da rebrota de cultivares e acessos de Panicum maximum Jacq. sob duas alturas de corte. Rev. Soc. Bras. Zoot. 29:660-668.

CONAGUA (2008) Comisión Nacional del Agua - Guerrero. Secretaria de Medio Ambiente y Recursos Naturales. Gobierno de México. 68 p.

Deregibus V A, R Sánchez A, J Casal J, M Telica J (1985) Tillering responses to enrichment of red light beneath the canopy in humid natural grassland. J. Appl. Ecol. 22:199-206.

Difante S G, D N Junior, S C Da Silva, B V P Euclides, M A Zanine, B Adese (2008) Dinâmica do perfilhamento do capim-marandu cultivado em duas alturas e três intervalos de corte. Rev. Bras. Zoot. 37:189-196.

Enríquez Q J F, F N Meléndez, E D A Bolaños (1999) Tecnología para la Producción y Manejo de Forrajes Tropicales en México. Libro Técnico Núm. 7. INIFAP. CIRGOC. Veracruz, México. 262 p.

García E (1981) Modificaciones al Sistema de Clasificación Climática de Köppen, para Adaptarlo a las Condiciones de la República Mexicana. UNAM. 4a ed. México, D. F. 271 p.

Hernández G A, C Matthew, J Hodgson (1997) Effect of spring management on perennial ryegrass and ryegrass-white clover pastures: 2. Tiller and growing point densities and population dynamics. New Zeal. J. Agric. Res. 40:37-50.

Hirata M, W Pakiding (2001) Tiller dynamics in a bahia grass (Paspalum notatum) pasture under cattle grazing. Trop. Grasslands 35:151160.

Lemaire G (2001) Ecophysiology of grasslands: dynamics aspects of forage plant populations in grazed swards. In: Proc. XIX Internat. Grassland Congress. 11-21 feb. São Pedro, São Paulo, Brasil. pp:2937.

Lemaire G, D Chapman (1996) Tissue flows in grazed plant communities. In: The Ecology and Management of Grazing Systems. J Hodgson, A W Illius (eds). Cab International. UK. pp:3-36.

Matthew C, S G Assuero, C K Black, N R Sackville Hamilton (2000) Tiller dynamics of grazed swards. In: Grassland Ecophysiology and Grazing Ecology. G Lemaire, J Hodgson, A Moraes, P C F Carvalho, C Nabinger (eds). CAB International. Wallingford, UK. pp:127-150.

Matthew C, C K Black, B M Butler (1993) Tiller dynamics of perennation in three herbage grasses. In: Proc. XIX Internat. Grassland Congress. 8-21 feb, New Zealand and Australia. pp:141-143.

Morais VR, D Fonseca M, D N Junior, J I Ribeiro J, J Fagundes L, M Moreira L, C Mistura, A Martuscello J (2006) Demografia de perfilhos basilares em pastagem de Brachiaria decumbens adubada com nitrogênio. Rev. Bras. Zoot. 35:380-388.

Ramírez R O, A Hernández G, S C Da Silva, J Pérez P, J F Enríquez Q, A R Quero C, J G Herrera H (2009) Acumulación de forraje, crecimiento y características estructurales del pasto Mombaza (Panicum maximum jacq.), cosechado a diferentes intervalo de corte. Téc. Pecu. Méx. 47:203-213.

Ramírez R O, A Hernández G, S C Da Silva, J Pérez P, S J De Souza J, R Castro R, F J Enríquez Q (2010) Características morfogénicas y su influencia en el rendimiento del pasto Mombaza, cosechado a diferentes intervalos de corte. Trop. Subtrop. Agroec. 12:303-311.

Santos M P, A M A Balsalobre, M Corsi (2004) Características morfogenéticas e taxa de acúmulo de forragem do capimMombaça submetido a três intervalos de pastejo. Rev. Bras. Zoot. 33:843-861.

Santos M P, M Corsi, A M A Balsalobre (1999) Efeito da freqüência de pastejo e da época do ano sobre a produção e a qualidade em Panicum maximum cvs. Tanzânia e Mombaça. Rev. Bras. Zoot. 28:244-249.

SAS Institute (2002) Statistical Analysis System (version 9.0 ed.). Cary NC, USA.

Skinner R H, J Nelson C (1994) Role of leaf appearance and the coleoptiles tiller in regulating tiller production. Crop Sci. 34:71-75.

Souza F H D (1999) Panicum maximum in Brasil. In: Forage Seed Production. 2. Tropical and Subtropical Species. D Loch S, J E Ferguson (eds). CAB. Publishing International pp:363-370.

Wolfinger R (1993) Covariance structure selection in general mixed models. Comm. Stat. Simul. 22:1079-1106. 